\title{
Correlation between Glucokinase Regulator (GCKR) Gene Polymorphisms and Serum Uric Acid Levels in Taiwanese Adolescents
}

Li-Ju Ho

Tri-Service General Hospital

Chieh-Hua Lu

Tri-Service General Hospital

Ruei-Yu Su

National Defense Medical Center

Fu-Huang Lin

National Defense Medical Center

Sheng-Chiang Su

Tri-Service General Hospital

Feng-Chih Kuo

Tri-Service General Hospital

Nain-Feng Chu

National Defense Medical Center

Yi-Jen Hung

National Defense Medical Center

Jhih-Syuan Liu

Tri-Service General Hospital

Chang-Hsun Hsieh ( $D$ 10324@yahoo.com.tw )

National Defense Medical Center

\section{Research Article}

Keywords: Hyperuricemia, glucokinase regulator, Taiwan, adolescent

Posted Date: July 26th, 2021

DOI: https://doi.org/10.21203/rs.3.rs-729848/v1

License: (1) (1) This work is licensed under a Creative Commons Attribution 4.0 International License.

Read Full License 


\section{Correlation between Glucokinase Regulator (GCKR) Gene Polymorphisms and}

\section{Serum Uric Acid Levels in Taiwanese Adolescents}

Li-Ju Ho ${ }^{1}$, Chieh-Hua Lu ${ }^{1}$, Ruei-Yu Su${ }^{2,3}$, Fu-Huang Lin ${ }^{4}$, Sheng-Chiang Su ${ }^{1}$, FengChih Kuo ${ }^{1}$, Nain-Feng Chu ${ }^{1,4}$, Yi-Jen Hung ${ }^{1}$, Jhih-Syuan Liu ${ }^{1 *}$, Chang-Hsun Hsieh ${ }^{1 * \pi}$

\section{Author Affiliations:}

${ }^{1}$ Division of Endocrinology and Metabolism, Department of Internal Medicine, TriService General Hospital, National Defense Medical Center, Taipei, Taiwan, R.O.C.;

${ }^{2}$ Division of Clinical Pathology, Department of Pathology, Tri-Service General Hospital, National Defense Medical Center, Taipei, Taiwan, R.O.C.;

${ }^{3}$ Department of Pathology and Laboratory Medicine, Taoyuan Armed Forces General Hospital, Taoyuan, Taiwan, R.O.C.;

${ }^{4}$ School of Public Health, National Defense Medical Center, Taipei, Taiwan, R.O.C.;

${ }^{*}$ Correspondence

I These authors contributed equally to this work.

\section{* Corresponding authors:}

\section{Chang-Hsun Hsieh, MD*}

Division of Endocrinology and Metabolism, Department of Internal Medicine, TriService General Hospital, National Defense Medical Center, Taipei, Taiwan, R.O.C.; Address: No.325, Section 2, Cheng-Kung Road, Neihu District, Taipei City, 11490, Taiwan, Republic of China. 
Tel: 886-2-87923311, Ext. 17129;

E-mail: 10324peter@gmail.com; 10324@,yahoo.com.tw

Jhih-Syuan, Liu, MD*

Division of Endocrinology and Metabolism, Department of Internal Medicine, Tri-

Service General Hospital, National Defense Medical Center, Taipei, Taiwan, R.O.C.;

Address: No.325, Section 2, Cheng-Kung Road, Neihu District, Taipei City, 11490, Taiwan, Republic of China.

Tel: 886-2-87923311, Ext. 17129;

Email: ajleonn21@hotmail.com.tw

Word count: Abstract, $\underline{1131}$ words; main text, $\underline{15,438}$ words.

Number of Tables \& Figures: Tables, $\underline{4}$; Figures, $\underline{0}$.

Keywords: Hyperuricemia, glucokinase regulator, Taiwan, adolescent 


\begin{abstract}
Background:

Recent genome-wide association studies revealed a positive association between hyperuricemia and variants of the glucokinase regulator (GCKR) gene in adults. This study replicated the genetic association in a Taiwanese adolescent population.
\end{abstract}

\title{
Methods:
}

The frequencies of different genotypes or alleles of the GCKR rs780094 and rs1260326 single-nucleotide polymorphism (SNP) were compared between 962 subjects (468 boys, 494 girls) with hyperuricemia (HUA) and normal uric acid (NUA) levels relative to gender. Logistic regression analysis was carried out to explore the genetic role in abnormal uric acid (UA) concentrations.

\section{Results:}

Boys had higher UA levels than girls $(6.68 \pm 0.06$ and $5.23 \pm 0.04 \mathrm{mg} / \mathrm{dl}$, respectively, $\mathrm{p}<0.001)$. The $\mathrm{T}$ allele had a higher HUA frequency than the $\mathrm{C}$ allele in both SNPs in girls; the rest of the frequencies of alleles and genotypes did not differentiate between subjects with HUA and NUA groups in both genders. However, after adjusting for confounding factors, the odds ratio for hyperuricemia incidence in TT genotype carriers was 1.75 (95\% confidence interval [CI]: 1.02 to 3.00$)$ and higher than the C-carrier genotype in rs1260326 of the girl population.

\section{Conclusion:}

The GCKR rs1260326 polymorphism is associated with higher UA concentrations in Taiwanese girl adolescents.

\section{Introduction}

Uric acid (UA) is a by-product of purine metabolism in humans. Hyperuricemia is associated with an increased risk of gout and is correlated to various chronic diseases such as hypertension, type 2 diabetes mellitus (T2DM), and cardiovascular 
disease (CVD) ${ }^{1,2}$. Moreover, hyperuricemia was shown to be an independent risk factor for all-cause and CVD mortality ${ }^{3}$. Therefore, it is important to evaluate the patients with hyperuricemia as early as possible.

Serum UA levels can be influenced by several lifestyle choices, such as protein and alcohol intake ${ }^{4}$. The variation of the prevalence of hyperuricemia may be attributed to the different lifestyle patterns. Genes also play an important role in serum UA concentrations ${ }^{5}$. Levels of serum UA are known to be heritable and related to mutations in genes encoding purine salvage pathway enzymes ${ }^{6}$. In recent decades, several case control and genome-wide association studies (GWAS) explored the role of genetic loci in hyperuricemia ${ }^{5,7-9}$. Most of these candidate genes regulate renal excretion of UA instead of influencing endogenous UA production.

Glucokinase (GCK) is a physiological glucose-sensor and playing a role in glucose-stimulated insulin release by phosphorylating glucose ${ }^{10}$. The glucokinase regulator (GCKR) gene is located on chromosome 2p23, contains 19 exons, and encodes a $68 \mathrm{kDa}$ protein ${ }^{11,12}$. Mutations in this gene are related to insulin signaling process related disorders. Recently, GWAS showed that common genetic variants of the GCKR gene were also associated with hyperuricemia and replicated among different ethnic populations, including rs $780094^{13-22}$ and $\mathrm{rs} 1260326^{13,21-24}$. However, not all the genetic variants of GCKR were associated with serum UA levels ${ }^{25,26}$. The genetic role of GCKR in serum UA concentrations remains to be defined.

Additionally, research has been done on adult populations, whose serum UA levels can easily be affected by lifestyle factors. Therefore, our present study explores the genetic association between common functional variants of the GCKR gene with serum UA concentrations in Taiwanese adolescents.

\section{Material and methods}

\section{Study sample and ethics statement}

Our study population was previously enrolled in an epidemiological study to evaluate the obesity and cardiovascular disease risk factors among school children in Taipei during 2006. The details of the study were published in previous works ${ }^{27,28}$. A total of 962 children ( 468 boys and 494 girls) were found to have available biochemical data and DNA samples were included in the final analyses. All methods were carried out in accordance with relevant guidelines and regulations. The Institutional Review 
Board of Tri-Service General Hospital approved this study (TSGHIRB No.095-05139, and TSGHIRB No.095-05-013), and the committee waived the need for written informed consent.

\section{Data collection and laboratory measurements}

In the previous study, all the participating subjects completed a questionnaire detailing their age, gender, demographic characteristics, personal history of diseases, pubertal development, and lifestyle characteristics, including cigarette smoking and alcohol consumption. Bodyweight (BW) was measured using a ruler attached to the scale by a trained nurse. Body mass index (BMI) was obtained through BW (kg) divided by the square of the height $(\mathrm{m})$. Blood samples were collected after a 10-hour fast and were used to determine plasma glucose and lipid profiles, including triglyceride (TG) and high-density lipoprotein cholesterol (HDL-C). The serum UA concentrations were measured using a uricase/peroxidase method (DVIA1650Autoanalyzer, Siemens Healthcare Diagnostics). Blood pressure (BP) was measured on the right arm after 10 minutes of rest in a seated position. Informed consent was obtained from the participating children and their parents before enrollment. The local Ethical Committee of the Scientific Institute approved the study.

\section{Definition of hyperuricemia and grouping}

The serum UA levels in children and adolescents change during development. Serum UA levels increase gradually from birth to the end of elementary school age, then rise dramatically in males and slightly in females, forming a considerable difference between the genders ${ }^{29}$. The definition of hyperuricemia is gender-specific with serum UA concentrations equal or greater to 7 $\mathrm{mg} / \mathrm{dl}$ for boys and $6 \mathrm{mg} / \mathrm{dl}$ for girls ${ }^{30}$. The study subjects were divided into two groups hyperuricemia (HUA) and normal uric acid (NUA) with gender specification.

\section{DNA extraction and genotyping}

DNA was isolated from blood samples using a QIAamp DNA Blood kit following the manufacturer's instructions (Qiagen) during the previous study. The 
qualities of isolated genomic DNAs were checked using agarose gel electrophoresis, and the quantities were determined using spectrophotometry. Genotyping was performed using TaqMan ${ }^{\circledR}$ Genotyping assays (Applied Biosystems Inc. (ABI)). TaqMan ${ }^{\circledR}$ PCR was performed according to the manufacturer's standard protocol. Each sample underwent 40 amplification cycles using a GeneAmp® PCR System 9700 instrument (ABI). Fluorescent signals of the two probes, corresponding to the detection of the two alleles, were analyzed using a PRISM ${ }^{\circledR} 7900$ HT Sequence Detection System (ABI). Genotypes were determined automatically by Sequence Detection Software (ABI). Two single-nucleotide polymorphisms (SNPs) within GCKR (rs1260326 and rs780094) were selected and validated for alleles and genotypes analyses according to previous studies ${ }^{13-26}$.

\section{Statistical analyses}

Analyses were performed using SPSS 20.0 statistical version (Chicago, IL, USA). The age, body weight, body height, BMI, demographic characteristics, and laboratory values were shown as mean and standard deviation (SD) and were compared between genders. The frequencies of alleles and genotypes were compared between groups of HUA and NUA. The relationships between dominant allele carrier and non-dominant allele carrier with contingency tables were also evaluated. The comparison was tested by Hardy-Weinberg equilibrium using a $\chi^{2}$ goodness-of-fit test. A logistic regression test was used to adjust for possible confounding factors, including age, BMI, and metabolic syndrome components. This test was also used to evaluate the independent role of genetic polymorphism on abnormal serum UA concentrations. The odds ratio (OR) with $95 \%$ confidence intervals for the risk genotypes was also calculated with a logistic regression test. All the above analyses were performed with gender specification. Statistical significance was defined as a pvalue less than 0.05 .

\section{Results}

Table 1 demonstrates the demographic characteristics of the study population. The mean age was $13.3 \pm 1.0$ years, and the mean BMI was $21.0 \pm 3.9 \mathrm{~kg} / \mathrm{m} 2$. Generally, boys had more unfavorable metabolic characteristics than girls. Serum UA 
levels were also higher in boys than girls $(6.68 \pm 0.06$ and $5.23 \pm 0.04 \mathrm{mg} / \mathrm{dl}$, respectively, $\mathrm{p}<0.001)$.

Table 1 Demographic characteristics of the study population

\begin{tabular}{|c|c|c|c|c|}
\hline Group & All (962) & Boys (468) & Girls (494) & $\mathrm{p}$ \\
\hline Age (years) & $13.3 \pm 1.0$ & $13.3 \pm 1.0$ & $13.4 \pm 1.0$ & 0.166 \\
\hline FPG (mg/dl) & $92.7 \pm 6.7$ & $93.8 \pm 6.4$ & $91.8 \pm 6.8$ & $<0.001$ \\
\hline BMI (Kg/m2) & $21.0 \pm 3.9$ & $21.6 \pm 4.2$ & $20.4 \pm 3.4$ & $<0.001$ \\
\hline $\mathrm{WC}(\mathrm{cm})$ & $76.4 \pm 9.8$ & $78.7 \pm 10.8$ & $74.3 \pm 8.2$ & $<0.001$ \\
\hline SBP (mmHg) & $114.8 \pm 13.7$ & $118.2 \pm 14.2$ & $111.6 \pm 12.4$ & $<0.001$ \\
\hline DBP (mmHg) & $69.7 \pm 10.0$ & $69.3 \pm 10.6$ & $70.0 \pm 9.4$ & 0.291 \\
\hline HDL-C (mg/dl) & $49.7 \pm 11.5$ & $47.5 \pm 11.3$ & $51.8 \pm 11.2$ & $<0.001$ \\
\hline TG (mg/dl) & $71.2 \pm 35.0$ & $70.9 \pm 38.9$ & $71.6 \pm 31.8$ & 0.112 \\
\hline \multirow[t]{2}{*}{ Uric acid } & & & $5.23 \pm 0.04$ & \\
\hline & $5.98 \pm 0.05$ & $6.68 \pm 0.06$ & & $<0.001$ \\
\hline$(\mathrm{mg} / \mathrm{dl})$ & & & & \\
\hline
\end{tabular}

FPG: fasting plasma glucose; BMI: body mass index; WC: waist circumference; SBP:

systolic blood pressure; DBP: diastolic blood pressure; HDL-C: high-density

lipoprotein cholesterol; TG: triglyceride

Table 2 shows the frequencies of alleles in different genders and different SNPs (rs780094 and rs1260326) between the HUA and NUA groups. It revealed that girls 
with HUA had a significantly higher T allele frequency than $\mathrm{C}$ allele in both SNPs (rs780094 and rs1260326).

Table 2. GCKR Alleles and abnormal uric acid frequencies in Taiwanese children

\begin{tabular}{|c|c|c|c|}
\hline & NUA n (\%) & HUA n $(\%)$ & $\mathrm{p}$ value \\
\hline \multicolumn{4}{|l|}{ Boys } \\
\hline GCKRrs1260326 & & & 0.102 \\
\hline $\mathrm{C}$ & $286(48.0 \%)$ & $147(42.5 \%)$ & \\
\hline $\mathrm{T}$ & $310(52.0 \%)$ & $199(57.5 \%)$ & \\
\hline GCKRrs780094 & & & 0.063 \\
\hline $\mathrm{C}$ & $302(50.5 \%)$ & $153(44.2 \%)$ & \\
\hline $\mathrm{T}$ & $296(49.5 \%)$ & $193(55.8 \%)$ & \\
\hline
\end{tabular}

Girls

GCKRrs 1260326

$0.028 *$

C

$418(51.0 \%) \quad 70(41.7 \%)$

$\mathrm{T}$

$402(49.0 \%) \quad 98(58.3 \%)$

GCKRrs780094

$0.034^{*}$

C

$431(52.4 \%) \quad 73(43.5 \%)$ 
NUA: normal UA; HUA: hyperuricemia

*Statistically significant differences, $\mathrm{p}<0.05$

There was no difference in genotype frequencies of both SNPs between the HUA and NUA groups in both genders (Table 3).

Table 3. GCKR genotypes and abnormal uric acid frequencies in Taiwanese children

$$
\text { NUA n }(\%) \quad \text { HUA n }(\%) \quad \text { p value }
$$

Boys

GCKRrs 1260326

0.188

$\mathrm{CC}$

$62(20.8 \%) \quad 25(14.5 \%)$

CT

$162(54.4 \%) \quad 97(56.1 \%)$

TT

$74(24.8 \%) \quad 51(29.5 \%)$

GCKRrs780094

0.13

$\mathrm{CC}$

$66(22.1 \%) \quad 27(15.6 \%)$

CT

$170(56.9 \%) \quad 99(57.2 \%)$

TT

$63(21.1 \%) \quad 47(27.2 \%)$

Girls 


$\begin{array}{lll}\text { CC } & 110(26.8 \%) & 16(19.0 \%) \\ \text { CT } & 198(48.3 \%) & 38(45.2 \%) \\ \text { TT } & 102(24.9 \%) & 30(35.7 \%)\end{array}$

NUA: normal UA; HUA: hyperuricemia

Table 4 illuminates the genetic dominant or recessive model on the impact of serum UA levels. Aside from rs1260326 in boys, subjects with TT genotypes had a higher risk of hyperuricemia compared to subjects with CC genotypes in both SNPs of both genders. However, after controlling for confounding factors, only the girl population remained statically significant, with an odds ratio (OR) of 2.29 (95\% confidence interval; CI: 1.11 to 4.73 ) for rs1260326 and OR of 2.28 (CI: 1.09 to 4.75) for rs780094. Furthermore, subjects with the TT genotype had a higher risk for hyperuricemia with an OR of 1.75 (CI: 1.02 to 3.00) than C carriers for rs 1260326 in the girl group.

Table 4. Logistic regression analyses of abnormal uric acid in Taiwanese children by GCKR gene genotypes (rs1260326、rs780094) 
Boys

\begin{tabular}{|c|c|c|}
\hline GCKRrs1260326 & OR $(95 \%$ CI $)$ & OR $(95 \% \mathrm{CI})$ \\
\hline CT vs. CC & 1.48 (0.88 to 2.52$)$ & $1.40(0.79$ to 2.48$)$ \\
\hline TT vs. CC & $1.71(0.95$ to 3.07$)$ & $1.56(0.82$ to 2.98$)$ \\
\hline TT vs $(\mathrm{CC}+\mathrm{CT})$ & $1.27(0.83$ to 1.92$)$ & $1.21(0.76$ to 1.93$)$ \\
\hline$(\mathrm{CT}+\mathrm{TT})$ vs $\mathrm{CC}$ & $1.56(0.94$ to 2.58$)$ & $1.45(0.84$ to 2.51$)$ \\
\hline GCKRrs780094 & OR $(95 \% \mathrm{CI})$ & OR $(95 \% \mathrm{CI})$ \\
\hline CT vs. CC & $1.42(0.85$ to 2.37$)$ & 1.34 (0.77 to 2.32$)$ \\
\hline TT vs. CC & $1.82(1.02$ to 3.28$) *$ & 1.65 (0.87 to 3.14$)$ \\
\hline TT vs $(\mathrm{CC}+\mathrm{CT})$ & $1.40(0.90$ to 2.16$)$ & $1.33(0.82$ to 2.15$)$ \\
\hline$(\mathrm{CT}+\mathrm{TT})$ vs $\mathrm{CC}$ & $1.53(0.94$ to 2.51$)$ & $1.42(0.83$ to 2.42$)$ \\
\hline
\end{tabular}

Girls

\begin{tabular}{lll} 
GCKRrs1260326 & OR $(95 \% \mathrm{CI})$ & OR $(95 \% \mathrm{CI})$ \\
CT vs. CC & $1.32(0.70$ to 2.47$)$ & $1.47(0.74$ to 2.91$)$ \\
TT vs. CC & $2.02(1.04$ to 3.93$) *$ & $2.29(1.11$ to 4.73$) *$ \\
TT vs $(\mathrm{CC}+\mathrm{CT})$ & $1.68(1.02$ to 2.76$) *$ & $1.75(1.02$ to 3.00$) *$ \\
& $1.56(0.87$ to 2.80$)$ & $1.74(0.92$ to 3.30$)$ \\
\hline
\end{tabular}




\begin{tabular}{|c|c|c|}
\hline GCKRrs780094 & OR $(95 \% \mathrm{CI})$ & OR $(95 \% \mathrm{CI})$ \\
\hline CT vs. CC & $1.49(0.80$ to 2.78$)$ & $1.70(0.87$ to 3.32$)$ \\
\hline TT vs. CC & $2.04(1.04$ to 4.00$) *$ & $2.28(1.09$ to 4.75$) *$ \\
\hline TT vs $(\mathrm{CC}+\mathrm{CT})$ & 1.55 (0.93 to 2.59$)$ & $1.58(0.91$ to 2.74$)$ \\
\hline$(\mathrm{CT}+\mathrm{TT})$ vs $\mathrm{CC}$ & $1.67(0.93$ to 3.00$)$ & $1.88(0.99$ to 3.56$)$ \\
\hline
\end{tabular}

Model 1: univariate analysis, Model 2: adjusted for age, BMI, waist circumference,

blood pressure, fasting plasma glucose, triglycerides, high density lipoprotein

cholesterol

OR: odds ratio; $\mathrm{CI}$ : confidence interval

*Statistically significant differences

\section{Discussion}

Hyperuricemia has a close correlation with several chronic disorders. While no prospective studies show an association between lower UA levels and prevention or risk reduction of CVD, patients with hyperuricemia should be monitored for CVD. Our study found that subjects with specific GCKR genetic polymorphisms had a higher risk for hyperuricemia with gender differences in Taiwanese adolescents. The subjects with this association could be prone to CVD in the future.

Many past studies explored the role of GCKR genetic variants on serum UA concentrations and/or gout. Most of these studies confirmed a strong correlation between genetic variants of GCKR polymorphism and hyperuricemia and/or development of gout, including rs $1260326^{13,21-24,26}$ and rs780094 ${ }^{13-22}$. Our report first 
demonstrates this association among a Taiwanese adolescent population, similarly to reports conducted in adult populations. Hyperuricemia is associated with insulin resistance ${ }^{31}$ and is considered one of the criteria of metabolic syndrome, indicating both diseases share a common genetic background. The close relationship between GCKR genetic variants and insulin resistance and/or glucose intolerance was explored in a previous study ${ }^{32}$. However, the exact mechanism of the GCKR genetic effect on uric acid concentrations remains unknown. Several possible mechanisms were proposed. Glucose metabolic abnormality related to GCK/GCKR expression leads to obesity, an important contributor to hyperuricemia ${ }^{33}$. A previous study raised a hypothesis that the GCKR gene might modulate hepatic inorganic phosphate (Pi) homeostasis and induce subsequent elevation of serum UA concentrations ${ }^{34}$. Hyperinsulinemia might cause a significant decrease in urinary UA excretion via increasing urate reabsorption in the kidney, inducing further hyperuricemia ${ }^{35}$. Another study demonstrated that a GCKR genetic variant was associated with lower fractional excretion of uric acid via increasing urate reabsorption in the proximal renal tubules ${ }^{23}$. Other research suggested that the genetic variant of GCKR influenced metabolite levels in the glycolytic pathway altering renal uric acid excretion ${ }^{36}$.

Nevertheless, not all the studies showed that GCKR genetic variants influenced uric acid concentrations ${ }^{25,26}$. The discrepancy in results may attribute to several factors. First, the study population and difference of minor allele frequency between ethnic populations may play a role. Second, limited sample sizes with limited power and inadequate effect sizes of the risk variants may also influence study results. Furthermore, the possible interaction between GCKR polymorphism and lifestyle patterns, such as drinking or eating habits and other genes, may also play a part, in which a European study showed that alcohol exposure with GCKR (rs780094) influenced the risk of hyperuricemia with a stronger role in the absence of alcohol ${ }^{17}$. This is consistent with the concept that GCKR controls gout risk through its physiologic role in glycolysis, presumably resulting in increased endogenous urate production. This gene-alcohol interaction was not observed in another Japanese study with the same polymorphism ${ }^{18}$. Genetic differences between ethnic populations may attribute to this discrepancy.

Interestingly, our study showed a gender difference in GCKR genetic variants on the influence of serum UA concentrations ( $\mathrm{T}$ allele). Only girls with TT genotypes among the two variants (rs780094 and rs1260326) had a higher risk for hyperuricemia 
than CC genotypes after adjusting for confounding factors. The data is limited about gender specification. No sexual dimorphism was observed in the GCKR genetic effect on uric acid levels in a Japanese study ${ }^{18}$ and a large scaled meta-analysis ${ }^{13}$. A recent study demonstrated significant sex-specific effect differences in the GCKR genetic variant (rs1260326), but the gender effect was not observed in the more stringent genome-wide test for SNP effects on urate levels ${ }^{37}$. The differences between results are unclear, but the study population, ethnic factor, and sex hormone may contribute. The prevalence of hyperuricemia or gout differed between genders, which might be attributed to the female sex hormone's uricosuric effect in the regulation of serum uric acid concentrations in animal and human studies ${ }^{38-40}$. SLC2A9 was also recognized as an apical urate transporter in the renal tubules ${ }^{41}$, which might increase the fractional excretion of uric acid and decrease serum uric acid concentrations ${ }^{42}$. Significant sexual dimorphism was also observed in the genetic variants of SLC2A9 in serum UA levels, implying a gender effect ${ }^{13,18}$. However, the exact mechanisms of gender difference on serum urate concentrations remain unclear ${ }^{43}$.

The current study's major strength was to explore the association between GCKR genetic variants and serum UA concentrations in a Taiwanese adolescent population with gender specification. There are still several limitations that need to be addressed. First, the definition of hyperuricemia is different between adults and adolescent populations. The information found here may not be applicable to adult populations. Second, many factors affect plasm UA levels, such as lifestyle patterns. Although the adolescent populations are assumed to have similar lifestyles, we did adjust for an alcohol consumption factor. Third, the relatively small numbers of our study subjects should be taken into consideration. Fourth, hormone profiles also influence plasma UA levels; we did not obtain hormone profiles during our analysis. Furthermore, complex diseases may be induced by multiple genetic interactions. The pleiotropy of the genetic functions may possibly explain the failure of replication to previous reports.

\section{Conclusions}

In conclusion, the present study showed that GCKR genetic polymorphisms influenced serum UA concentrations with gender differences in Taiwanese adolescents. However, larger future studies are required to confirm this association. 


\section{Author contributions}

Conceptualization: Nain-Feng Chu, Chang-Hsun Hsieh

Data curation: Li-Ju Ho, Ruei-Yu Su, Nain-Feng Chu, Yi-Jen Hung

Formal analysis: Fu-Huang Lin, Nain-Feng Chu

Methodology: Feng-Chih Kuo, Sheng-Chiang Su, Chieh-Hua Lu, Fu-Huang Lin

Project administration: Yi-Jen Hung, Jhih-Syuan Liu, Chang-Hsun Hsieh

Resources: Nain-Feng Chu, Jhih-Syuan Liu, Chang-Hsun Hsieh

Software: Li-Ju Ho, Ruei-Yu Su, Chieh-Hua Lu, Nain-Feng Chu, Fu-Huang Lin

Supervision: Chang-Hsun Hsieh, Jhih-Syuan Liu,

Writing - original draft: Li-Ju Ho

Writing - review \& editing: Li-Ju Ho, Ruei-Yu Su, Chang-Hsun Hsieh

\section{Acknowledgments}

All the authors thank the Enago academic website for their expertise and assistance throughout all aspects of our study and for their help in writing assistance and language editing of our manuscript.

\section{Funding}

This study was funded by Tri-Service General Hospital Research and Medical Affairs Bureau, Ministry of National Defense Foundation (TSGH-C105-119 to ChangHsun Hsieh, and TSGH-C108-144 to Jhih-Syuan Liu). The two funders play important roles in the study design, data collection and analysis, decision to publish, and preparation of the manuscript.

\section{References}

1 So, A. \& Thorens, B. Uric acid transport and disease. J Clin Invest 120, 17911799, doi:10.1172/jci42344 (2010).

2 Feig, D. I., Kang, D. H. \& Johnson, R. J. Uric acid and cardiovascular risk. $N$ Engl J Med 359, 1811-1821, doi:10.1056/NEJMra0800885 (2008). 
3 Chen, J. H., Chuang, S. Y., Chen, H. J., Yeh, W. T. \& Pan, W. H. Serum uric acid level as an independent risk factor for all-cause, cardiovascular, and ischemic stroke mortality: a Chinese cohort study. Arthritis Rheum 61, 225232, doi:10.1002/art.24164 (2009).

4 Sugie, T., Imatou, T., Miyazaki, M. \& Une, H. The effect of alcoholic beverage type on hyperuricemia in Japanese male office workers. J Epidemiol 15, 41-47, doi:10.2188/jea.15.41 (2005).

5 Nath, S. D. et al. Genome scan for determinants of serum uric acid variability. $J$ Am Soc Nephrol 18, 3156-3163, doi:10.1681/asn.2007040426 (2007).

6 Riches, P. L., Wright, A. F. \& Ralston, S. H. Recent insights into the pathogenesis of hyperuricaemia and gout. Hum Mol Genet 18, R177-184, doi:10.1093/hmg/ddp369 (2009).

7 Wei, W. et al. Characterisation of genome-wide association epistasis signals for serum uric acid in human population isolates. PLoS One 6, e23836, doi:10.1371/journal.pone.0023836 (2011).

8 Cummings, N. et al. Genome-wide scan identifies a quantitative trait locus at 4p15.3 for serum urate. Eur J Hum Genet 18, 1243-1247, doi:10.1038/ejhg.2010.97 (2010).

9 Wallace, C. et al. Genome-wide association study identifies genes for biomarkers of cardiovascular disease: serum urate and dyslipidemia. $A m J$ Hum Genet 82, 139-149, doi:10.1016/j.ajhg.2007.11.001 (2008).

10 Matschinsky, F. M., Glaser, B. \& Magnuson, M. A. Pancreatic beta-cell glucokinase: closing the gap between theoretical concepts and experimental realities. Diabetes 47, 307-315, doi:10.2337/diabetes.47.3.307 (1998).

11 Warner, J. P., Leek, J. P., Intody, S., Markham, A. F. \& Bonthron, D. T. Human glucokinase regulatory protein (GCKR): cDNA and genomic cloning, complete primary structure, and chromosomal localization. Mamm Genome 6, 532-536, doi:10.1007/bf00356171 (1995).

12 Veiga-da-Cunha, M. et al. Mutations in the glucokinase regulatory protein gene in 2p23 in obese French caucasians. Diabetologia 46, 704-711, doi:10.1007/s00125-003-1083-y (2003).

13 Kolz, M. et al. Meta-analysis of 28,141 individuals identifies common variants within five new loci that influence uric acid concentrations. PLoS Genet 5, e1000504, doi:10.1371/journal.pgen.1000504 (2009). 
14 van der Harst, P. et al. Replication of the five novel loci for uric acid concentrations and potential mediating mechanisms. Hum Mol Genet 19, 387395, doi:10.1093/hmg/ddp489 (2010).

15 Sun, X. et al. Serum uric acid levels are associated with polymorphisms in the SLC2A9, SF1, and GCKR genes in a Chinese population. Acta Pharmacol Sin 35, 1421-1427, doi:10.1038/aps.2014.87 (2014).

16 Phipps-Green, A. J. et al. Twenty-eight loci that influence serum urate levels: analysis of association with gout. Ann Rheum Dis 75, 124-130, doi:10.1136/annrheumdis-2014-205877 (2016).

17 Rasheed, H., Stamp, L. K., Dalbeth, N. \& Merriman, T. R. Interaction of the GCKR and A1CF loci with alcohol consumption to influence the risk of gout. Arthritis Res Ther 19, 161, doi:10.1186/s13075-017-1369-y (2017).

18 Takeuchi, F. et al. Genetic impact on uric acid concentration and hyperuricemia in the Japanese population. J Atheroscler Thromb 20, 351-367, doi:10.5551/jat.15727 (2013).

19 Wang, J. et al. Association between gout and polymorphisms in GCKR in male Han Chinese. Hum Genet 131, 1261-1265, doi:10.1007/s00439-0121151-9 (2012).

20 Zhou, Z. W. et al. Polymorphisms in GCKR, SLC17A1 and SLC22A12 were associated with phenotype gout in Han Chinese males: a case-control study. BMC Med Genet 16, 66, doi:10.1186/s12881-015-0208-8 (2015).

21 Dong, Z. et al. Effects of multiple genetic loci on the pathogenesis from serum urate to gout. Sci Rep 7, 43614, doi:10.1038/srep43614 (2017).

22 Yang, Q. et al. Multiple genetic loci influence serum urate levels and their relationship with gout and cardiovascular disease risk factors. Circ Cardiovasc Genet 3, 523-530, doi:10.1161/circgenetics.109.934455 (2010).

23 Köttgen, A. et al. Genome-wide association analyses identify 18 new loci associated with serum urate concentrations. Nat Genet 45, 145-154, doi:10.1038/ng.2500 (2013).

24 Matsuo, H. et al. Genome-wide association study of clinically defined gout identifies multiple risk loci and its association with clinical subtypes. Ann Rheum Dis 75, 652-659, doi:10.1136/annrheumdis-2014-206191 (2016).

25 Stark, K. et al. Common polymorphisms influencing serum uric acid levels contribute to susceptibility to gout, but not to coronary artery disease. PLoS One 4, e7729, doi:10.1371/journal.pone.0007729 (2009). 
26 Li, Z. et al. Replication of Gout/Urate Concentrations GWAS Susceptibility Loci Associated with Gout in a Han Chinese Population. Sci Rep 7, 4094, doi:10.1038/s41598-017-04127-4 (2017).

27 Chu, N. F., Rimm, E. B., Wang, D. J., Liou, H. S. \& Shieh, S. M. Clustering of cardiovascular disease risk factors among obese schoolchildren: the Taipei Children Heart Study. Am J Clin Nutr 67, 1141-1146, doi:10.1093/ajen/67.6.1141 (1998).

28 Chu, N. F., Rimm, E. B., Wang, D. J., Liou, H. S. \& Shieh, S. M. Relationship between anthropometric variables and lipid levels among school children: The Taipei Children Heart Study. Int J Obes Relat Metab Disord 22, 66-72, doi:10.1038/sj.ijo.0800546 (1998).

29 Kubota, M. Hyperuricemia in Children and Adolescents: Present Knowledge and Future Directions. J Nutr Metab 2019, 3480718, doi:10.1155/2019/3480718 (2019).

30 Kliegman, R., Arvin, A. M. \& Behrman, R. E. (Saunders, Philadelphia, 1997).

31 Liu, X. Z., Xu, X., Zhu, J. Q. \& Zhao, D. B. Association between three noninsulin-based indexes of insulin resistance and hyperuricemia. Clin Rheumatol 38, 3227-3233, doi:10.1007/s10067-019-04671-6 (2019).

32 Onuma, H. et al. The GCKR rs780094 polymorphism is associated with susceptibility of type 2 diabetes, reduced fasting plasma glucose levels, increased triglycerides levels and lower HOMA-IR in Japanese population. $J$ Hum Genet 55, 600-604, doi:10.1038/jhg.2010.75 (2010).

33 Suhre, K. et al. Human metabolic individuality in biomedical and pharmaceutical research. Nature 477, 54-60, doi:10.1038/nature10354 (2011).

34 Arden, C. et al. Elevated glucose represses liver glucokinase and induces its regulatory protein to safeguard hepatic phosphate homeostasis. Diabetes 60, 3110-3120, doi:10.2337/db11-0061 (2011).

35 Quiñones Galvan, A. et al. Effect of insulin on uric acid excretion in humans. Am J Physiol 268, E1-5, doi:10.1152/ajpendo.1995.268.1.E1 (1995).

36 Narang, R. K. et al. Population-specific factors associated with fractional excretion of uric acid. Arthritis Res Ther 21, 234, doi:10.1186/s13075-0192016-6 (2019). 
37 Tin, A. et al. Target genes, variants, tissues and transcriptional pathways influencing human serum urate levels. Nat Genet 51, 1459-1474, doi:10.1038/s41588-019-0504-x (2019).

38 Mumford, S. L. et al. Serum uric acid in relation to endogenous reproductive hormones during the menstrual cycle: findings from the BioCycle study. Hum Reprod 28, 1853-1862, doi:10.1093/humrep/det085 (2013).

39 Yahyaoui, R. et al. Effect of long-term administration of cross-sex hormone therapy on serum and urinary uric acid in transsexual persons. $J$ Clin Endocrinol Metab 93, 2230-2233, doi:10.1210/jc.2007-2467 (2008).

40 Eraly, S. A. et al. Multiple organic anion transporters contribute to net renal excretion of uric acid. Physiol Genomics 33, 180-192, doi:10.1152/physiolgenomics.00207.2007 (2008).

41 Sato, Y. et al. The case for uric acid-lowering treatment in patients with hyperuricaemia and CKD. Nat Rev Nephrol 15, 767-775, doi:10.1038/s41581019-0174-z (2019).

42 Le, M. T., Shafiu, M., Mu, W. \& Johnson, R. J. SLC2A9--a fructose transporter identified as a novel uric acid transporter. Nephrol Dial Transplant 23, 2746-2749, doi:10.1093/ndt/gfn349 (2008).

43 DeBosch, B. J., Kluth, O., Fujiwara, H., Schürmann, A. \& Moley, K. Earlyonset metabolic syndrome in mice lacking the intestinal uric acid transporter SLC2A9. Nat Commun 5, 4642, doi:10.1038/ncomms5642 (2014). 


\section{Supplementary Files}

This is a list of supplementary files associated with this preprint. Click to download.

- GCKRdraftfinalV1table1.docx

- GCKRdraftfinalV1table2.docx

- GCKRdraftfinalV1table3.docx

- GCKRdraftfinalV1table4.docx 